\title{
Inhibitory Motor Control in Response Stopping and Response Switching
}

\author{
Naomi M. Kenner, ${ }^{1}$ Jeanette A. Mumford, ${ }^{2}$ Rebecca E. Hommer, ${ }^{3}$ Martha Skup, ${ }^{4}$ Ellen Leibenluft, ${ }^{5}$ \\ and Russell A. Poldrack $\mathbf{k}^{1,2,6}$ \\ ${ }^{1}$ Department of Psychology, University of California, Los Angeles, Los Angeles, California 90095-1563, 2Department of Psychology, University of Texas at \\ Austin, Austin, Texas 78712, ${ }^{3}$ Yale University Child Study Center, and ${ }^{4}$ Biostatistics Division in School of Public Health, Yale University, New Haven, \\ Connecticut 06529, ${ }^{5}$ Section on Bipolar Spectrum Disorders, Mood and Anxiety Disorders Program, National Institute of Mental Health, Bethesda, \\ Maryland 20892-2670, and 'Department of Neurobiology, University of Texas at Austin, Austin, Texas 78712
}

While much is known about the neural regions recruited in the human brain when a dominant motor response becomes inappropriate and must be stopped, less is known about the regions that support switching to a new, appropriate, response. Using functional magnetic resonance imaging with two variants of the stop-signal paradigm that require either stopping altogether or switching to a different response, we examined the brain systems involved in these two forms of executive control. Both stopping trials and switching trials showed common recruitment of the right inferior frontal gyrus, presupplementary motor area, and midbrain. Contrasting switching trials with stopping trials showed activation similar to that observed on response trials (where the initial response remains appropriate and no control is invoked), whereas there were no regions that showed significantly greater activity for stopping trials compared with switching trials. These results show that response switching can be supported by the same neural systems as response inhibition, and suggest that the same mechanism of rapid, nonselective response inhibition that is thought to support speeded response stopping can also support speeded response switching when paired with execution of the new, appropriate, response.

\section{Introduction}

In order for goal-directed behavior to remain appropriate in a changing environment, the brain must support processes that countermand behaviors which become unsuitable. These processes might involve a single nonselective inhibitory "emergency brake" that prevents all responses and is recruited for any form of response inhibition, or there might be different neural mechanisms suited to different inhibitory situations. When a response must not only be stopped but also rapidly switched to a newly appropriate response, a nonselective mechanism could be less efficient than a selective mechanism that prevents only specific responses while leaving others unaffected, whether by an dedicated mechanism for selective inhibition or by general mechanisms of biased competition in the frontal cortex. The goal of this study is to compare the neural regions involved in response stopping to those recruited during response switching.

In functional magnetic resonance imaging (fMRI) studies, response inhibition is repeatedly associated with activity in the right inferior frontal gyrus (RIFG) (Konishi et al., 1999; Chevrier et al., 2007; Xue et al., 2008). Lesions (Aron et al., 2003) and transcranial magnetic stimulation (TMS) (Chambers et al., 2006,

Received Feb. 26, 2010; revised April 21, 2010; accepted May 12, 2010.

This work was supported by a grant from the James S. McDonnell Foundation to R.A.P., a National Science Foundation graduate fellowship to N.M.K., and the National Institute of Mental Health Intramural Research Program.

Correspondence should be addressed to Naomi M. Kenner, Department of Psychology, University of California, Los Angeles, 1285 Franz Hall, Box 951563, Los Angeles, CA 90095-1563. E-mail: nmk@ucla.edu.

DOI:10.1523/JNEUROSCI.1096-10.2010

Copyright $\odot 2010$ the authors $\quad 0270-6474 / 10 / 308512-07 \$ 15.00 / 0$
2007) of RIFG reduce performance. Converging evidence from fMRI, lesion, and TMS studies also implicates medial prefrontal regions in response inhibition (Garavan et al., 1999; Li et al., 2006; Chevrier et al., 2007), particularly the presupplementary motor area (pre-SMA) (Aron and Poldrack, 2006; Floden and Stuss, 2006; Chen et al., 2009). The subthalamic nucleus (StN) has also been linked to performance in the stop signal task (Aron and Poldrack, 2006; Eagle et al., 2008; Li et al., 2008). Human diffusion tensor imaging demonstrates anatomical connectivity between these three regions (Aron et al., 2007). This potential network may support rapid nonselective stopping via the hyperdirect pathway of the basal ganglia (Aron et al., 2007), which induces rapid extensive inhibition of the basal ganglia (Nambu et al., 2002).

Selective response control may also recruit this nonselective pathway (Coxon et al., 2009). Alternatively, it may recruit frontostriatal circuitry that allows for selective modulation of the basal ganglia (Mink, 1996), or it could be achieved by mechanisms of biased competition in the cortex (Desimone and Duncan, 1995; O'Reilly et al., 1999; Miller and Cohen, 2001; O'Reilly, 2006) in which competing responses or goals are resolved cortically by interactions, such as local inhibition and feedback circuits through subcortical regions, between the neurons coding for each response option. If the neural regions comprising the putative hyperdirect pathway are active for response stopping but not switching, this would indicate that switching recruits a different mechanism for response control. If they are active in both situations it would suggest that a common mechanism can be recruited to achieve both types of control.

The present fMRI study compares neural activity during speeded response countermanding in a stop-signal task, which is 


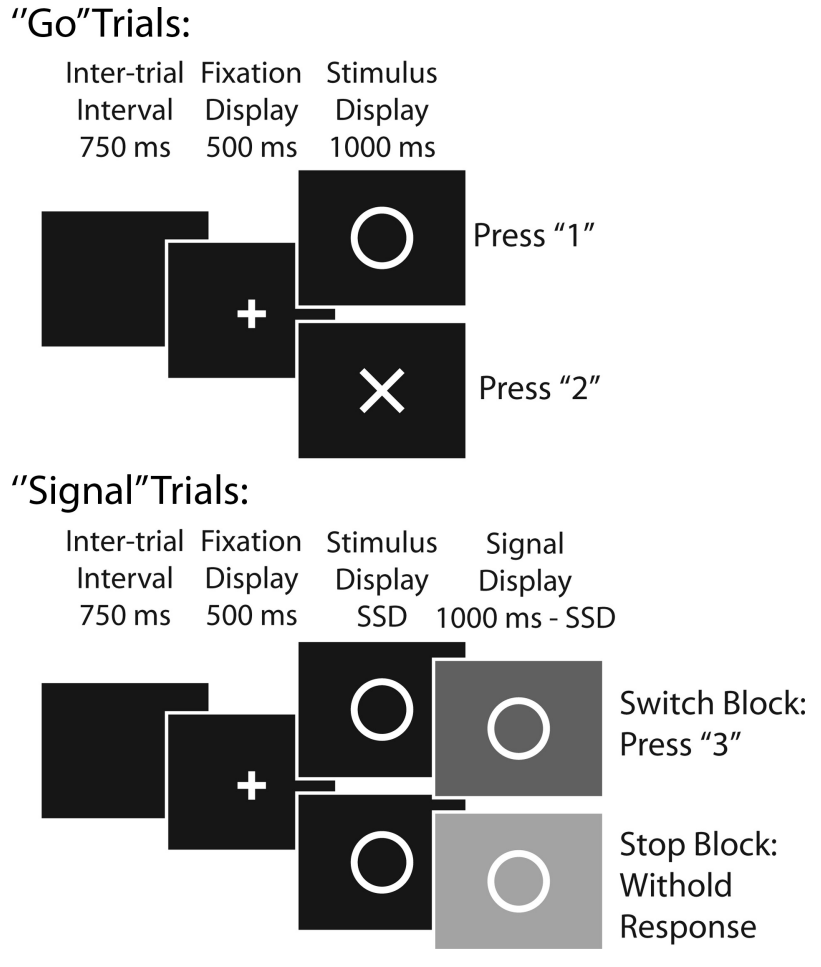

Figure 1. Time course and components of the behavioral task. Of the trials with stimuli, $2 / 3$ were go trials and $1 / 3$ were signal trials. Signal trials featured both " $X$ " and " 0 " stimuli.

well suited for a fast, nonselective, inhibitory mechanism, to neural activity during speeded response switching in a switch-signal task, which is more suited to a selective inhibitory mechanism or biased competition in the cortex.

\section{Materials and Methods}

\section{Participants}

Healthy adults between the ages of 22 and 40 were recruited via advertisement. Psychiatric health was determined by the Structured Clinical Interview for DSM Disorders (SCID) diagnostic interview with an addendum for attention deficit hyperactivity disorder (ADHD), administered by a clinician. Participants had no history of psychiatric illness, either past or present, including ADHD. Psychiatric illness in first degree relatives was not exclusionary. Good physical health was evaluated by history and physical exam. Participants were not on prescription medications, and had not taken over-the-counter medications for at least $48 \mathrm{~h}$ before being scanned. Participants gave written informed consent, and the study was approved by the National Institute of Mental Health Institutional Review Board; data were shared with University of California, Los Angeles for analysis under an approved data sharing agreement.

Exclusion criteria were set a priori: participants were excluded from analysis if scans had inadequate quality, or if participants had poor task performance $(<65 \%$ correct on go trials) or excessive movement (translational displacement $>2.5 \mathrm{~mm}$ in any plane). Thirty-nine adults were scanned, and 15 were excluded from analysis: two for poor performance, two for discontinuing performance early, seven for excessive movement, two due to equipment malfunction, and two for excess artifact. The resulting sample size analyzed was 24 with $54 \%$ male and an average age of 29.8 years $( \pm 6.0 \mathrm{SD})$.

\section{Task}

Participants performed a stopping task (Fig. 1) that was adapted from previous published work (Logan et al., 1997). On each trial, participants viewed a white fixation cross on a black background for $500 \mathrm{~ms}$, at which point the fixation cross was replaced by an "X" or an "O" for $1000 \mathrm{~ms}$. An intertrial interval of $750 \mathrm{~ms}$ followed the stimulus presentation. Participants responded during stimulus presentation by pressing " 1 " on a but- ton box if "X" appeared, and "2" if the stimulus was "O". They were told to respond as quickly as possible and in less than a second, unless a "signal" occurred. Signals followed about a third of the stimulus onsets, and consisted of a background color change. If the background changed to red participants were instructed to try to withhold their response, and if the background became blue, participants switched their response to " 3 ", regardless of the stimulus currently displayed. These two types of signal trials, stopping and switching, were blocked such that only one type of signal was presented during each run of trials. Participants were told which type of signal trial they would get at the start of each run. Each run consisted of 44 go-trials, 20 signal-trials, and 22 blank-trials (no stimulus onset or signal) that were presented in a random order.

During the first stop and switch signal trials, the signal was presented $500 \mathrm{~ms}$ after the "X" or "O" appeared on the screen. The delay between the stimulus onset and signal [stop or switch signal delay (SSD)] on subsequent signal trials was determined by the success of the stopping or switching performance on the previous signal trial. For example, if a participant in a switching run successfully switched responses on a switch trial, the SSD for the next switch trial would be increased by $50 \mathrm{~ms}$, making switching more difficult. If the participant failed to switch, however, the SSD for the next switch trial would be reduced by $50 \mathrm{~ms}$, making switching easier. This staircasing procedure drove participants toward $50 \%$ successful signal trial performance.

Participants received training on the task before entering the scanner. After each block of trials, participants received feedback on their mean go-trial reaction time (goRT) for that block and were instructed to speed up their responding if their mean goRT was $>1000 \mathrm{~ms}$. Slow-responding participants also received this feedback while scanning, but it was typically only necessary during the training phase. Training was given until mean goRT was $<1000 \mathrm{~ms}$.

\section{Data acquisition}

Scanning took place in a General Electric Signa 3 Tesla MRI system at the National Institutes of Health. Images were presented using Avotec Silent Vision Glasses, which were placed directly above participants' eyes. Head movement was restricted by use of foam padding. Sagittal localization and manual shimming were performed before the acquisition of functional data using an echoplanar imaging (EPI) sequence. EPI images were obtained with single shot gradient echo $\mathrm{T}^{*}$ weighting in 23 contiguous 5 $\mathrm{mm}$ axial slices per brain volume, positioned parallel to the anterior commissure-posterior commissure line. Imaging parameters were as follows: $64 \times 64$ matrix; TR (repetition time $)=2000 \mathrm{~ms}$; TE $($ echo time $)=$ $40 \mathrm{~ms} ; 240 \mathrm{~mm}$ field of view; voxel size $3.75 \times 3.75 \times 5 \mathrm{~mm}$.

All imaging data for each participant were acquired in a single scanning session, during which the participant completed eight runs of EPI image acquisition while performing the task. Each run consisted of 86 trials, of which 44 were go-trials, 20 were signal-trials, and 22 were null events (fixation only). The signal-trials were stop-signal for half the runs and switch-signal for the other runs. Signal type was alternated in pairs, such that a participant might receive runs in one of the following two orders: stop-stop-switch-switch-stop-stop-switch-switch, or switchswitch-stop-stop-switch-switch-stop-stop. The order was randomly selected for each participant. The three trial types (go, signal, fixation) were presented in a randomized order in each run for each participant.

Additionally, a high-resolution T1-weighted anatomical image was obtained using a standardized magnetization-prepared gradient echo sequence (1801 mm sagittal slices; field of view $=256$; number of excitations $=1 ; \mathrm{TR}=11.4 \mathrm{~ms} ; \mathrm{TE}=4.4 \mathrm{~ms} ;$ matrix $=256 \times 256 ; \mathrm{TI}=300$ ms; bandwidth $=130 \mathrm{~Hz} /$ pixel, $33 \mathrm{kHz} / 256$ pixels). This image was used to aid spatial normalization.

\section{Data analysis}

Behavioral data. Behavior was evaluated during scan acquisition by measurements of reaction time on go trials (goRT), percentage accuracy on go trials, and percentage success on signal trials (successful stopping for stop runs, successful switching for switch runs). Linear regression was used to assess the ability of age to predict each of the measurements. The durations of the theorized control processes [stop signal reaction time (SSRT) for stop runs and switch signal reaction time (SCRT) for switch 


\section{Table 1. Behavioral task results}

\begin{tabular}{lccc}
\hline & Stopping & Switching & Error \\
\hline Go trial accuracy & $88 \%$ & $86 \%$ & $1.1 \%$ \\
Signal trial accuracy & $60 \%$ & $40 \%$ & $2.1 \%$ \\
Average reaction time, correct go trials (ms) & 788 & 773 & 6.5 \\
Estimates of signal reaction time (ms) & SSRT $=193$ & SCRT $=260$ & 8.4 \\
\hline
\end{tabular}

runs] are typically estimated by subtracting the SSD at which stopping or switching is at chance (i.e., 50\% stopping or switching success) from the median correct goRT (Logan et al., 1997; Williams et al., 1999). Although the SSD staircasing was intended to drive participants' signal-trial success to $50 \%$ and keep it there, actual performance tended to deviate slightly from this level. An interpolation algorithm was therefore adopted to calculate SSRT and SCRT: the mean SSD for the task (stopping or switching) is subtracted from the goRT at the $x$ th percentile of the distribution of correct go trial RTs for that task, where $x$ is the participant's signal-trial failure rate for that task (i.e., percentage failed stopping or failed switching) (Logan, 1994).

Imaging data. The FSL software toolbox [Oxford Centre for Functional Magnetic Resonance Imaging of the Brain (FMRIB)] was used to perform preprocessing and statistical analysis of the imaging data (Smith et al., 2004). Motion correction was accomplished with MCFLIRT (Jenkinson et al., 2002). Independent components analysis was performed on each run using MELODIC (Beckmann and Smith, 2004) and components reflecting motion or other artifacts were identified (Tohka et al., 2008). Denoised datasets were created by removing the artifactual components from each run. FILM prewhitening, high-pass filtering with a cutoff period of $80 \mathrm{~s}$, and spatial smoothing (full-width half-maximum) with a Gaussian kernel of $6 \mathrm{~mm}$ were applied to the images. Statistical analyses at the single-participant level were performed using a general linear model with FSL's FEAT (FMRI Expert Analysis Tool), using triallevel events (correct go, incorrect go, successful signal, failed signal) and their temporal derivatives as regressors, following convolution with a canonical double-gamma hemodynamic response function. The contrasts estimated included: correct go-baseline, failed go-baseline, successful signal-baseline, failed signal-baseline, successful signal-correct go, failed signal-correct go, and successful signal-failed signal. All first level contrast estimates were spatially normalized to the Montreal Neurological Institute (MNI)-152 template using FSL's registration tool, FLIRT, using 7 DOF to align the functional image to the structural image, and 12 DOF to align the structural image to the MNI152 (Jenkinson et al., 2002). A second-level analysis was used to compute task-level contrasts (stopping runs, switching runs, and switching runs-stopping runs) for each participant, collapsed across runs and treating runs as a fixed effect. Top-level mixed effects analyses were performed for each trial-level contrast using FSL's FLAME module (FMRIB's local analysis of mixed effects) (Smith et al., 2004), thresholded $Z$ (Gaussianized T/F) statistic maps were created with an uncorrected cluster-forming threshold of $z>$ 2.3 , and significance of the remaining clusters was determined using cluster-based Gaussian random field theory, controlling familywise error across the entire brain at $p=0.05$ (Friston et al., 1994).

\section{Results}

\section{Behavioral data}

Behavioral results are summarized in Table 1. Error for each measure is computed as a within-subject confidence interval (Loftus and Masson, 1994). Accuracy on go-trials (i.e., percentage correct classification) was high for both the stopping and switching versions of the task. The rate of success on the signaltrials was near the intended level of 50\% (60\% for stopping and $40 \%$ for switching). There was no difference in average correct go trial reaction times between the correct go trials in the stopping task block and the correct go trials in the switching task block. The estimated duration of the switch process (SCRT) took, on average, $67 \mathrm{~ms}$ longer than the estimated duration of the stop process (SSRT) [paired $t$ test: $t_{(23)}=5.7, p<0.05$ ]. This differ- ence between SSRT and SCRT is an artifact of the way accuracy was calculated. To be considered a successful switch, the switch action ("press 3") had to be detected during the $1000 \mathrm{~ms}$ response window. On a third of the switch trials classified as failed $(20 \%$ of all switch block signal trials), the switch action was detected in the $750 \mathrm{~ms}$ intertrial interval. This inflated failure rate increased the value drawn from the distribution of correct goRTs at the failure rate percentile, which resulted in longer SCRT estimates. When we correct for this by reclassifying accuracy to include the signal trials with switch responses during the intertrial interval, average SCRT drops to $208 \mathrm{~ms}$ and is statistically equivalent to the average SSRT. The difference between the SSRT and SCRT therefore cannot be interpreted. There was no effect of age on any behavioral measurement.

\section{Imaging data}

\section{Whole brain contrasts}

Cluster coordinates for all reported contrasts are provided in the supplemental material (available at www.jneurosci.org).

The go process: correct go-baseline. The go process, or stimulusdependent response selection and execution, was analyzed by comparing the correct go trials to baseline. For both the stopping and switching tasks, correct go-baseline showed significant positive activation in left motor cortex, and bilateral supplementary motor area (SMA), putamen, thalamus, midbrain, right frontal pole, and occipital cortex (Fig. 2). Direct comparisons between correct go for stopping and switching (correct go stopping-correct go switching) showed no differences, suggesting that the go process involves a similar set of regions in both tasks. This interpretation is supported by a conjunction analysis of the go trials during the stopping task and the switching task, performed by multiplying the binarized and thresholded (whole-brain corrected) statistical maps for the two conditions (Nichols et al., 2005).

Stopping and switching: successful signal-correct go. The processes supporting both stopping and switching can be estimated from the successful signal-correct go contrast, which is based on the race model of response inhibition (Logan and Cowan, 1984; Band et al., 2003; Boucher et al., 2007), in which stopping and going processes are simultaneously engaged and race toward completion after a stop signal. According to this model, a successful stopping trial involves both go processes and stopping processes and therefore contrasting stopping trials with go trials provides the best isolation of effective stopping processes. In contrast, comparison between successful and unsuccessful stop trials would not isolate the stopping process (since it occurs on both types of trials, according to the model), but instead would incorporate differences in the go process (because, according to the race model (Logan and Cowan, 1984), failed stop trials are also due to faster go processes), along with processes related to the detection of error and conflict. For both the stopping (Fig. $3 \mathrm{~A}$ ) and switching (Fig. 3B) tasks, the successful signal-correct go contrast showed positive activation in bilateral lateral prefrontal cortex (PFC), IFG, insula, orbitofrontal cortex (OFC), caudate, pallidum, midbrain, medial temporal lobe (MTL), amygdala, and occipital regions.

The switching-stopping contrast (Fig. 3C) showed positive bilateral differences in OFC, putamen, pallidum, temporoparietal junction (TPJ), motor cortex, and posterior parietal cortex. Left hemisphere differences were seen in the caudate, thalamus, and SMA. Right hemisphere differences were seen in the IFG. No negative activity was observed in the switching-stopping contrast 


\section{A Stopping Task:}

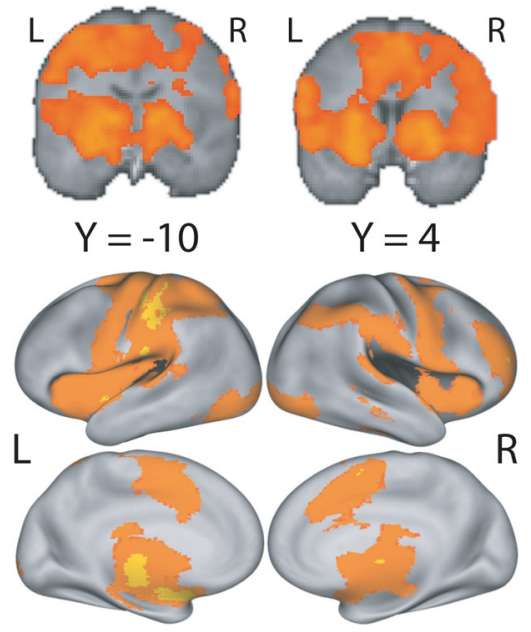

0
B Switching Task:
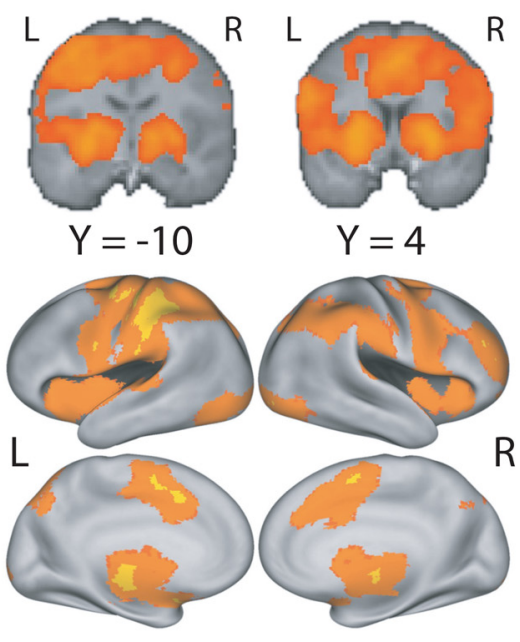

10

\section{Switching + Stopping:}
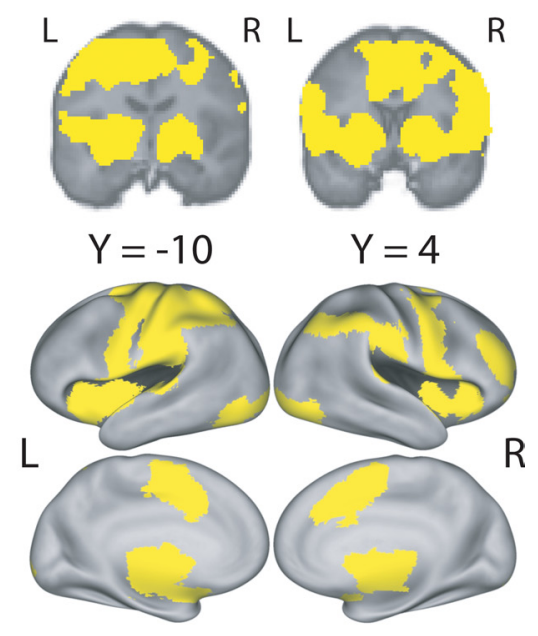

Figure 2. Correct go-baseline: positive neural activity associated with the contrast of correct go-baseline for stopping task go trials $(\boldsymbol{A})$, switching task go trials ( $\boldsymbol{B})$, and the conjunction of stopping task go trials and switching task go trials (C).

(data not shown), which is equivalent to saying there was no above-threshold positive activity in stopping-switching.

To identify common regions of activity during signal trials of the stopping and switching tasks, conjunction analyses were performed as described above. For the successful signal-baseline contrasts (data not shown), both stopping and switching showed positive activity in bilateral frontal pole, dorsolateral PFC, insula, IFG, thalamus, basal ganglia, midbrain, anterior cingulate cortex (ACC), pre-SMA, SMA, occipital and superior parietal cortices. Left-lateralized common activity was seen in motor cortex. Although it may seem surprising that motor cortex is active during successfully stopped trials, it is consistent with the race model's idea that the go process and stop process are active in parallel when inhibition is attempted (Logan and Cowan, 1984), and such activity has been previously observed (Aron and Poldrack, 2006).

For the successful signal-correct go contrasts, conjunction analysis of tasks (Fig. 3D) showed that both tasks had positive activity in bilateral frontal pole, IFG, insula, pre-SMA, occipital, superior parietal, and inferior temporal regions. Right-lateralized activity was seen in the striatum, midbrain, amygdala, and hippocampus. These findings demonstrate that the regions consistently engaged during response inhibition are also engaged by response switching.

To determine the effects of age, an additional model using age as a covariate was run for each contrast described above (correct go-baseline and successful signal-correct go). The patterns of activation described above were also observed using the model that included age as a covariate. Thus, it appears that age is not a significant factor in the present results.

Switching beyond stopping: conjunction with going. One way of modeling the difference between switching and stopping in this task is simply that switching involves a stop process for the old response along with a go process for the new response. If this is the case, it would be expected that the additional positive activity observed during the switch task signal trials when compared with the stop task signal trials would be due to going. If so, the positive activation from the comparison of the difference between switch- ing and stopping between successful signal and correct go

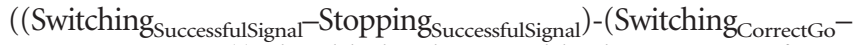
Stopping CorrectGo $\left._{0}\right)$ ) should closely resemble the contrast of correct go-baseline.

Positive activity common to these two contrasts is seen in left motor cortex, pre-SMA, thalamus, and basal ganglia (Fig. 4). Similarities between these contrasts support the idea that switching in this task is equivalent to a stopping process plus a go process for the new response.

\section{Discussion}

This study provides evidence that a set of neural regions thought to support rapid and nonselective response inhibition is involved in both speeded stopping and switching. Switching additionally involved regions consistent with response selection and execution. These findings are consistent with the idea that response switching can be supported by a stopping process, such as a nonselective inhibitory mechanism implemented by the hyperdirect pathway, paired with a go process for the new response.

According to the race model of response inhibition (Logan and Cowan, 1984), when an initiated behavior becomes inappropriate a stop process is triggered and runs in parallel with the go process, and the process that reaches completion first drives behavior. During a signal-trial in the stop- or switch-signal tasks, the race model claims that both stop- and go-processes are present. To isolate stop-process activity in the BOLD signal, the go-process is removed by subtracting correct go-trial activity from successful signal-trial activity. This contrast also removes perceptual, cognitive, and motor processes that are sufficient for correct response selection and execution in the go-trial. Using this contrast, the present study shows activity in regions that are consistent with the putative hyperdirect pathway between inferior frontal cortex and the subthalamic nucleus both when responses are withheld and when they are switched. Conjunction analysis between these tasks using this contrast show that these tasks share regional activation (Fig. 3D), encompassing the regions previously associated with response inhibition (Konishi et al., 1999; Aron and Poldrack, 2006; Li et al., 2006, 2008; Chevrier 


\section{A Stopping Task:}
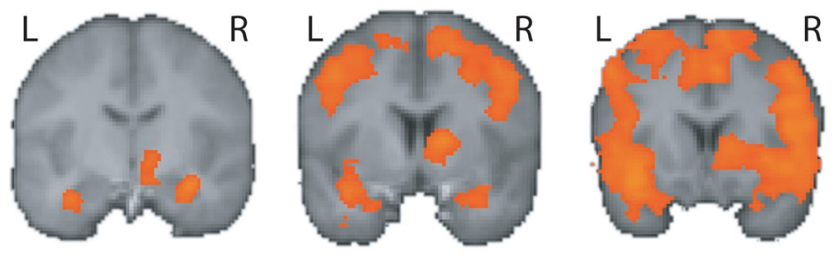

$\mathrm{Y}=-10$

$Y=4$

$Y=14$

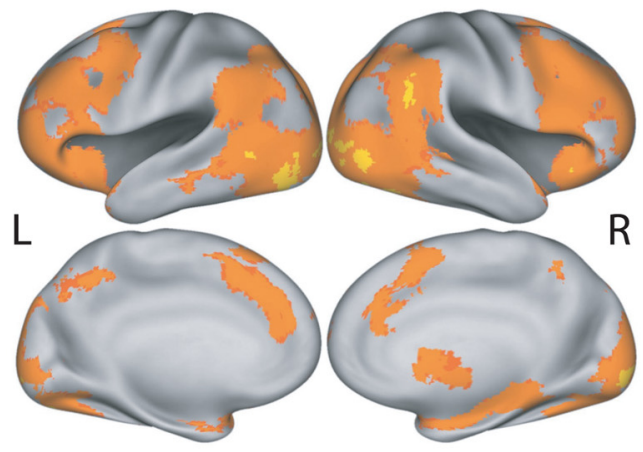

0

\section{Switching - Stopping:}

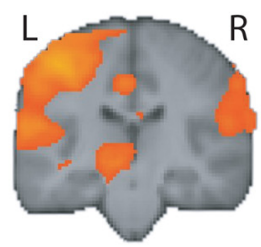

$Y=-22$
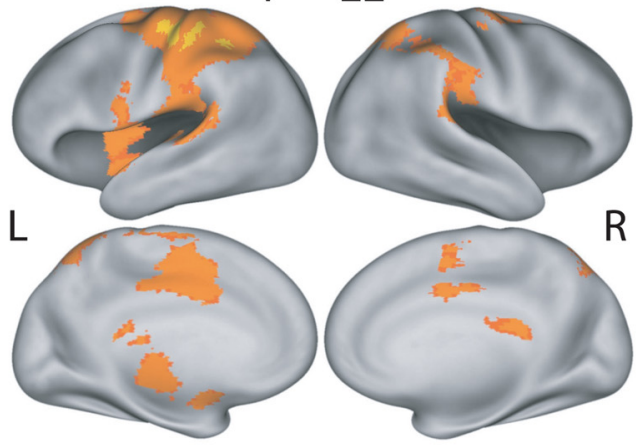

0

\section{B Switching Task:}
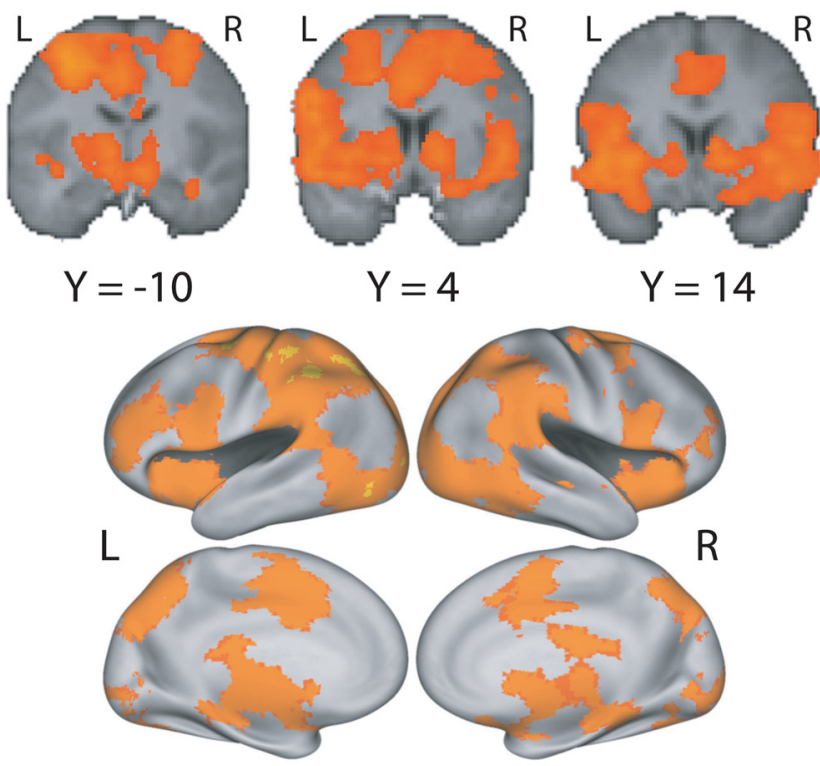

0

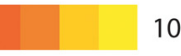

D Switching + Stopping:
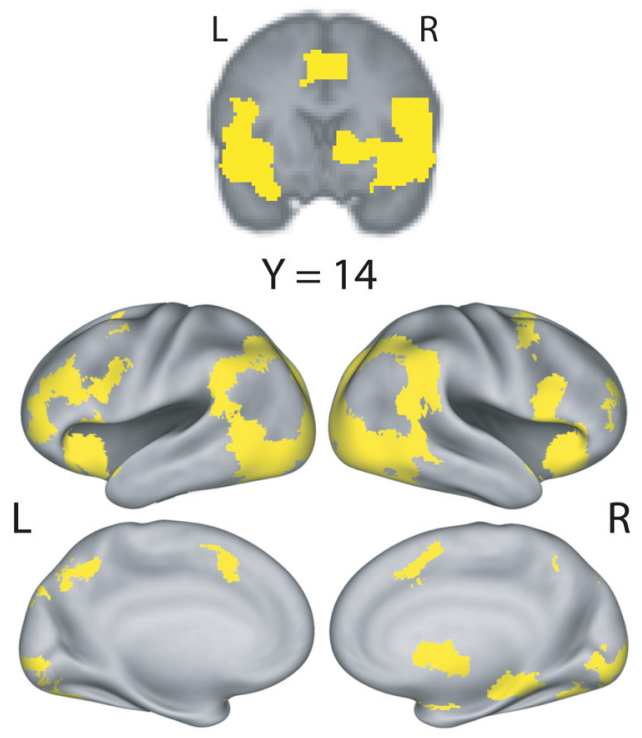

Figure 3. Successful signal-correct go: positive neural activity associated with the contrast of successful signal-correct go for stopping task trials $(\boldsymbol{A})$, switching task trials $(\boldsymbol{B})$, the difference between the tasks, switching task trials-stopping task trials $(\boldsymbol{C})$, and the similarities between the tasks, switching task trials + stopping task trials $(\boldsymbol{D})$.

et al., 2007; Xue et al., 2008) and tasks with both stopping and switching components (Konishi et al., 1999; Aron et al., 2004). Regions differing between these tasks using this contrast were only seen for the switch-task, and are consistent with regions that support a go-process (Fig. 4). While these results show that neural processes involved in response switching are consistent with a model of nonselective inhibition (Initial Go + Nonselective Stop + New Go), they do not rule out alternative models of selective inhibitory mechanisms (Initial Go + Selective Stop + New Go), or biased cortical competition (Initial Go $+\mathrm{New}$ Go). A selective mechanism might show involvement of specialized subcortical regions (i.e., activity in regions that modulate the Initial Go response, but not regions that affect other motor responses) but because the responses in our task were three right-hand key presses these would involve adjacent neural regions that are unlikely to be distinguishable at the acquired resolution. A task that required switching between regions that are not mapped near each other (for example, a movement of the tongue and of the toes) would be better suited to address this question of selectivity. If switching is accomplished through biased competition in the prefrontal representations of the action-goals, activity seen in subcortical regions might reflect the effects of cortical conflict on relevant 


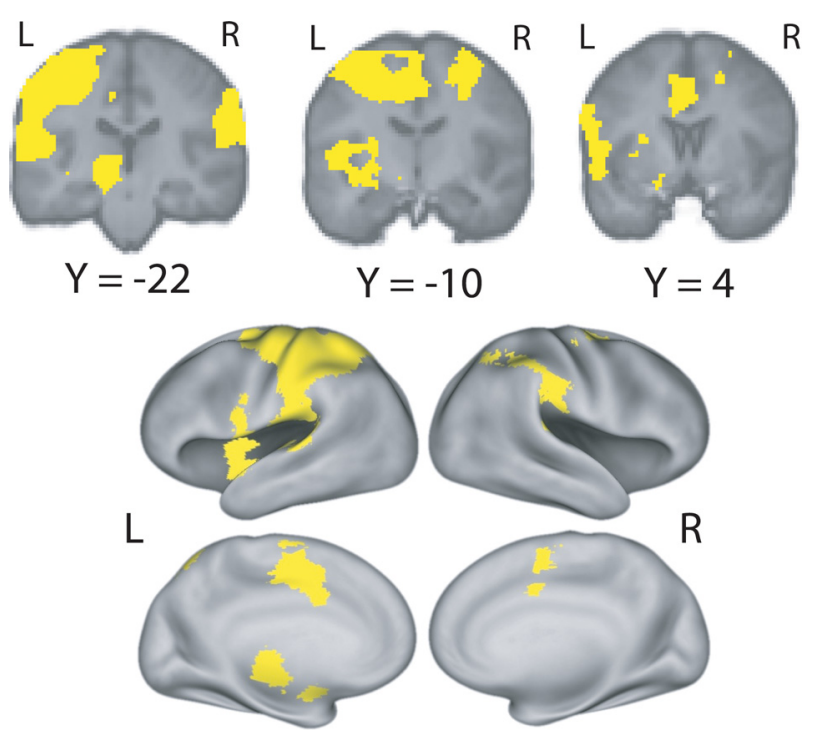

Figure 4. Switching-stopping is similar to going: positive neural activity associated with the

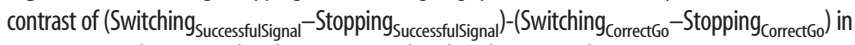
conjunction with activity related to correct go- baseline during switching.

processes in these regions, such as response selection mechanisms, rather than the effects of an active inhibitory mechanism implemented through the hyperdirect pathway. The current results show activity in inferior prefrontal regions for switching and stopping that is not seen during the Initial Go. Because this activity would not be expected for the Initial Go or New Go processes this could be interpreted as evidence against the biased competition model and support a specific role for IFG in an active inhibitory mechanism during response switching.

Recent behavioral work (Coxon et al., 2007; Aron and Verbruggen, 2008; Verbruggen et al., 2008) has questioned the combinations of processes that might support switching by modeling the behavioral measures that would be consistent with possible models. Their results were inconsistent with a model of Initial Go + New Go (Verbruggen et al., 2008) and, together, these studies suggest that speeded response switching can be successfully modeled as a primary go process followed by a nonselective stop process followed by (or simultaneous with) a secondary response process (Initial Go + Nonselective Stop + New Go). The results presented here are consistent with the idea that the neural processes supporting speeded response switching can follow this model.

While the contrast of successful signal-correct go attempts to isolate the inhibitory process from other processes including motor, perceptual, and cognitive, some may play a relatively stronger role in supporting signal trials than go trials and remain reflected in the BOLD signal. Both go trials and signal trials involve perceiving the same stimuli, but signal trials contain an additional visual component (a change in background color). Switch trials also involve interpretation of the signal as another cue that must be attended and associated cognitively with a motor response. Consistent with these extra processing needs, activity is present in occipital regions for the contrast of successful signal-correct go for both tasks and for the conjunction of tasks (Fig. 3), and in the TPJ for successful signal-correct go. This activity is seen for both tasks compared with baseline (Fig. $3 A, B$ ), for switching-stopping (Fig. 3C), and for the conjunction of tasks (Fig. 3D). This pattern of temporal activity was previously thought to reflect auditory processing of a tone used as the stop signal, but current findings show a similar pattern without an auditory signal (see supplemental material for a conjunction analysis between auditory and visual signals in a stop signal task). While the TPJ has recently been linked to inhibition (Nakata et al., 2008), this region has been strongly implicated in visual attention, particularly in stimulus-driven orienting (Corbetta and Shulman, 2002; Kincade et al., 2005; Hahn et al., 2006), in the context of goal relevance and control (Kincade et al., 2005; Yeh et al., 2007).

Processes supporting the detection of conflict and error may also be active to a greater degree during signal trials than nonsignal trials. The presence of a signal should trigger response conflict detection in addition to response inhibition, and this process should also survive the contrast of successful signal-correct go. Additionally, the rare signals could trigger a ramping up of proactive control in the form of conflict monitoring processes. These processes of performance monitoring and control have been associated with medial prefrontal regions, particularly the ACC (Botvinick et al., 2001). In the stop signal task, medial frontal BOLD activity has been associated with error-related processes beyond those that contribute to corrective control ( $\mathrm{Li}$ et al., 2006). BOLD activity observed in the pre-SMA and SMA in the selective-stopping condition of an adaptive stopping paradigm was suggested to support resolution of preresponse conflict and response adjustment (Coxon et al., 2009). The present study observed medial frontal activity in both tasks during the successful signal-correct go contrast, which may reflect response-control processes beyond inhibition as this study was not designed to pull these apart. The insula has also been implicated in error-related processes in the stop signal task (Ramautar et al., 2006), and activity in this structure has been linked to error processing but not post-error response control ( $\mathrm{Li}$ et al., 2006). While the present study did not attempt to isolate error-related processing, insula activity was observed in the contrast of successful signalcorrect go during both stopping and switching and could reflect this processing.

In conclusion, the present study provides evidence that regions consistent with the hyperdirect pathway, which may serve nonselective response inhibition, are involved both when a response is stopped and when it is switched. Regions observed during response selection and execution are also active during response switching, suggesting that the primary neural difference between stopping and switching in this task is a process supporting the appropriate new response. One interpretation of these results is that the hyperdirect pathway can support both response inhibition and response switching, and that response switching can be achieved by nonselective response inhibition. The results may be specific to the characteristics of the response switching scenario in this task, and situations with different characteristics may require different processes to support switching and recruit different neural systems. In the present study, characteristics that may have influenced the selectivity of the inhibition process include the importance put on the speed of response switching, the fact that the switch-response was always the same, and the similarity of the go-response and switch-response. The interpretation of this study is limited by the similarity of the Initial and New responses, and by the inability to model New Go responses in isolation from signal trials. While the present results fit the idea that a common inhibitory process is engaged for different types of control, it is possible that other sets of neural regions could be recruited in different inhibitory situations to support, for example, more selective response inhibition, or that activity being attributed here to an inhibitory mechanism could be serving a more general role in cortical competition. While a number of 
questions remain, the present study demonstrates that switching a response can be modeled using the same nonselective response inhibition process that has been successfully used to model stopping a response.

\section{References}

Aron AR, Poldrack RA (2006) Cortical and subcortical contributions to stop signal response inhibition: role of the subthalamic nucleus. J Neurosci 26:2424-2433.

Aron AR, Verbruggen F (2008) Stop the presses: dissociating a selective from a global mechanism for stopping. Psychol Sci 19:1146-1153.

Aron AR, Fletcher PC, Bullmore ET, Sahakian BJ, Robbins TW (2003) Stopsignal inhibition disrupted by damage to right inferior frontal gyrus in humans. Nat Neurosci 6:115-116.

Aron AR, Monsell S, Sahakian BJ, Robbins TW (2004) A componential analysis of task-switching deficits associated with lesions of left and right frontal cortex. Brain 127:1561-1573.

Aron AR, Behrens TE, Smith S, Frank MJ, Poldrack RA (2007) Triangulating a cognitive control network using diffusion-weighted magnetic resonance imaging (MRI) and functional MRI. J Neurosci 27:3743-3752.

Band GPH, van der Molen MW, Logan GD (2003) Horse-race model simulations of the stop-signal procedure. Acta Psychol (Amst) 112:105-142.

Beckmann CF, Smith SM (2004) Probabilistic independent component analysis for functional magnetic resonance imaging. IEEE Trans Med Imaging 23:137-152.

Botvinick MM, Braver TS, Barch DM, Carter CS, Cohen JD (2001) Conflict monitoring and cognitive control. Psychol Rev 108:624-652.

Boucher L, Palmeri TJ, Logan GD, Schall JD (2007) Inhibitory control in mind and brain: an interactive race model of countermanding saccades. Psychol Rev 114:376-397.

Chambers CD, Bellgrove MA, Stokes MG, Henderson TR, Garavan H, Robertson IH, Morris AP, Mattingley JB (2006) Executive "brake failure" following deactivation of human frontal lobe. J Cogn Neurosci 18:444-455.

Chambers CD, Bellgrove MA, Gould IC, English T, Garavan H, McNaught E, Kamke M, Mattingley JB (2007) Dissociable mechanisms of cognitive control in prefrontal and premotor cortex. J Neurophysiol 98:3638-3647.

Chen CY, Muggleton NG, Tzeng OJ, Hung DL, Juan CH (2009) Control of prepotent responses by the superior medial frontal cortex. Neuroimage 44:537-545.

Chevrier AD, Noseworthy MD, Schachar R (2007) Dissociation of response inhibition and performance monitoring in the stop signal task using event-related fMRI. Hum Brain Mapp 28:1347-1358.

Corbetta M, Shulman GL (2002) Control of goal-directed and stimulusdriven attention in the brain. Nat Rev Neurosci 3:201-215.

Coxon JP, Stinear CM, Byblow WD (2007) Selective inhibition of movement. J Neurophysiol 97:2480-2489.

Coxon JP, Stinear CM, Byblow WD (2009) Stop and go: the neural basis of selective movement prevention. J Cogn Neurosci 21:1193-1203.

Desimone R, Duncan J (1995) Neural mechanisms of selective visual attention. Annu Rev Neurosci 18:193-222.

Eagle DM, Baunez C, Hutcheson DM, Lehmann O, Shah AP, Robbins TW (2008) Stop-signal reaction-time task performance: role of prefrontal cortex and subthalamic nucleus. Cereb Cortex 18:178-188.

Floden D, Stuss DT (2006) Inhibitory control is slowed in patients with right superior medial frontal damage. J Cogn Neurosci 18:1843-1849.

Friston K, Worsley K, Frackowiak R (1994) Assessing the significance of focal activations using their spatial extent. Hum Brain Mapp 1:210-220.

Garavan H, Ross TJ, Stein EA (1999) Right hemispheric dominance of inhibitory control: an event-related functional MRI study. Proc Natl Acad Sci U S A 96:8301-8306.

Hahn B, Ross TJ, Stein EA (2006) Neuroanatomical dissociation between bottom-up and top-down processes of visuospatial selective attention. Neuroimage 32:842-853.
Jenkinson M, Bannister P, Brady M, Smith S (2002) Improved optimization for the robust and accurate linear registration and motion correction of brain images. Neuroimage 17:825-841.

Kincade JM, Abrams RA, Astafiev SV, Shulman GL, Corbetta M (2005) An event-related functional magnetic resonance imaging study of voluntary and stimulus-driven orienting of attention. J Neurosci 25:4593-4604.

Konishi S, Nakajima K, Uchida I, Kikyo H, Kameyama M, Miyashita Y (1999) Common inhibitory mechanism in human inferior prefrontal cortex revealed by event-related functional MRI. Brain 122:981-991.

Li CS, Huang C, Constable RT, Sinha R (2006) Imaging response inhibition in a stop-signal task: neural correlates independent of signal monitoring and post-response processing. J Neurosci 26:186-192.

Li CS, Yan P, Sinha R, Lee TW (2008) Subcortical processes of motor response inhibition during a stop signal task. Neuroimage 41:1352-1363.

Loftus G, Masson M (1994) Using confidence intervals in within-subject designs. Psychon Bull Rev 1:476-490.

Logan G (1994) On the ability to inhibit thought and action: a users' guide to the stop signal paradigm. In: Inhibitory processes in attention, memory, and language (Dagenbach D, Carr TH, eds), pp 189-239. San Diego: Academic.

Logan G, Cowan W (1984) On the ability to inhibit thought and action: a theory of an act of control. Psychol Rev 91:295-327.

Logan G, Schachar R, Tannock R (1997) Impulsivity and inhibitory control. Psychol Sci 8:60-64.

Miller EK, Cohen JD (2001) An integrative theory of prefrontal cortex function. Annu Rev Neurosci 24:167-202.

Mink JW (1996) The basal ganglia: focused selection and inhibition of competing motor programs. Prog Neurobiol 50:381-425.

Nakata H, Sakamoto K, Ferretti A, Gianni Perrucci M, Del Gratta C, Kakigi R, Luca Romani G (2008) Somato-motor inhibitory processing in humans: an event-related functional MRI study. Neuroimage 39:1858-1866.

Nambu A, Tokuno H, Takada M (2002) Functional significance of the corticosubthalamo-pallidal 'hyperdirect' pathway. Neurosci Res 43:111-117.

Nichols T, Brett M, Andersson J, Wager T, Poline JB (2005) Valid conjunction inference with the minimum statistic. Neuroimage 25:653-660.

O'Reilly R, Braver T, Cohen J (1999) A biologically based computational model of working memory. In: Models of working memory: mechanisms of active maintenance and executive control (Miyake A, Shah P, eds), pp 375-411. New York: Cambridge UP.

O’Reilly RC (2006) Biologically based computational models of high-level cognition. Science 314:91-94.

Ramautar JR, Slagter HA, Kok A, Ridderinkhof KR (2006) Probability effects in the stop-signal paradigm: the insula and the significance of failed inhibition. Brain Res 1105:143-154.

Smith SM, Jenkinson M, Woolrich MW, Beckmann CF, Behrens TEJ, Johansen-Berg H, Bannister PR, Luca MD, Drobnjak I, Flitney DE, Niazy RK, Saunders J, Vickers J, Zhang Y, Stefano ND, Brady JM, Matthews PM (2004) Advances in functional and structural MR image analysis and implementation as FSL. Neuroimage 23 [Suppl 1]:S208-S219.

Tohka J, Foerde K, Aron AR, Tom SM, Toga AW, Poldrack RA (2008) Automatic independent component labeling for artifact removal in fMRI. Neuroimage 39:1227-1245.

Verbruggen F, Schneider DW, Logan GD (2008) How to stop and change a response: the role of goal activation in multitasking. J Exp Psychol Hum Percept Perform 34:1212-1228.

Williams BR, Ponesse JS, Schachar RJ, Logan GD, Tannock R (1999) Development of inhibitory control across the life span. Dev Psychol 35:205-213.

Xue G, Aron AR, Poldrack RA (2008) Common neural substrates for inhibition of spoken and manual responses. Cereb Cortex 18:1923-1932.

Yeh YY, Kuo BC, Liu HL (2007) The neural correlates of attention orienting in visuospatial working memory for detecting feature and conjunction changes. Brain Res 1130:146-157. 